\title{
Conformational changes in inter- $\alpha$-trypsin inhibitor heavy chain 4 activate its tumor-specific activity in mice with B16 melanoma
}

\author{
NATALYA G. KORMOSH ${ }^{1}$, TATYANA V. DAVIDOVA ${ }^{1}$, VLADIMIR N. KOPYLTSOV ${ }^{2}$, \\ MARINA V. SEREBRYAKOVA ${ }^{3,4}$, AIGULO.KABIEVA ${ }^{5}$, KONSTANTIN E. VOYUSHIN ${ }^{1}$, SURIYA M. SITDIKOVA ${ }^{1}$, \\ BERICK S. AMANDZHOLOV ${ }^{1}$, MICHAIL V. KISELEVSKII ${ }^{1}$ and FEDOR V. DONENKO ${ }^{1}$ \\ ${ }^{1}$ Laboratory of Cell Immunity, Institute of Diagnosis and Therapy, N.N. Blokhin Russian Cancer Research Center, \\ Moscow 115478; ${ }^{2}$ Research Institute for Physical-Chemical Medicine, Moscow 119828; ${ }^{3}$ Institute of Gene Biology, \\ Russian Academy of Sciences, Moscow 117334; ${ }^{4}$ Moscow State University, Moscow 119991, Russia; \\ ${ }^{5}$ Kazakh Scientific Research Institute of Oncology and Radiobiology, Ministry of Health, \\ Republic of Kazakhstan, Almaaty 480012, Kazakhstan
}

Received September 7, 2014; Accepted May 28, 2015

DOI: $10.3892 / \mathrm{mmr} .2015 .3961$

\begin{abstract}
It is known that blood serum proteins of tumor-bearing mice display tumor-specific activity. However, to date, the nature of this activity has remained elusive, and no tumor-specific proteins have been detected in the blood serum of tumor-bearing animals compared with those in healthy animals. The present study postulated and investigated the hypothesis that the observed tumor-specific activity of the blood serum proteins is not associated with the appearance of novel serum proteins but with changes in the conformation of the existing ones. The present study showed conformational changes of two serum albumin proteins and inter- $\alpha$-trypsin inhibitor heavy chain 4 (ITIH4) in mice with B16 melanoma compared to tumor-free mice, as determined by differences in the products of proteolysis by proteomic analysis following column chromatography. The differences in the conformation of serum albumin in mice with B16 melanoma and tumor-free mice were accompanied by a change in the interaction of these molecules with the fatty acid spin probe 16-doxyl stearic acid. The differential conformation of ITIH4 in mice with B16 melanoma and that in tumor-free mice was accompanied by inhibition of tumor growth and increased life span. Analysis of the role of protease-anti-proteases (serpins) in the serum of tumor-bearing animals in tumor growth confirmed the hypothesis that tumor growth in the body is mediated, at least in part, via balancing of serpins.
\end{abstract}

Correspondence to: Professor Dr Fedor Vitaliyevich Donenko, Laboratory of Cell Immunity, Institute of Diagnosis and Therapy, N.N. Blokhin Russian Cancer Research Center, 24 Kashirskoye Shosse, Moscow 115478, Russia

E-mail: fedor.donenko@gmail.com

Key words: tumor growth, serpin, melanoma B16

\section{Introduction}

A previous study by our group demonstrated changes in the glycosylation of serum proteins in tumor-bearing mice (1). However, it was not possible to establish a link between the various $\mathrm{N}$-linked oligosaccharide residues and the tumors of animals or cancer patients (1-3). Another previous study by our group showed that the serpin $\alpha$-anti-trypsin from blood serum inhibits or blocks the growth of tumors (4). The question that arises from these studies is how proteins in the blood serum of tumor-bearing mice as well as in the blood serum of tumor-free mice acquire tumor-associated biological activity. It is known that the blood serum of tumor-bearing animals contains a certain factor which specifically accelerates tumor growth $(2,5)$. However, all attempts to identify this factor as a novel protein in the blood serum of tumor-bearing animals were unsuccessful $(5,6)$. Significant progress has been made toward comprehensive protein expression profiling, and numerous biomarker candidates have been identified; however, none of the reported biomarkers have been proven to be beneficial for patients with cancer (7). It is therefore necessary to identify these factors through their biological activity in vivo. The above-mentioned $\mathrm{N}$-linked oligosaccharide residues are part of protein molecules. Changes in these residues [in the previous study by our group they became shorter (4)] are likely to lead to conformational changes of the protein molecule. It is can therefore be assumed that changes in the glycosylation of proteins occur in order to modify the conformation of the protein molecule $(8,9)$. Conformational changes of a protein lead to changes in its biological activity. In organisms displaying tumor growth, no novel proteins were detected in the blood serum compared with those in tumor-free organisms; this may be due to the existing proteins undergoing conformational changes leading to the activation of their tumor-associated activity (10).

The most informative method for studying proteins is proteomic analysis. This analysis involves, in particular, the proteolysis of proteins. It is known that certain proteolytic 
enzymes have specific cleavage sites. These sites should fulfill the following requirements: They are required to contain certain amino acids, and furthermore, these sites should be available for their specific cleavage enzymes. If the proteolysis of a protein isolated from the serum of a tumor-bearing mouse and that of the same protein isolated from the serum of a tumor-free mouse results in products, it is indicated that this protein has a differential conformation between the tumor-bearing and the tumor-free mouse. However, until recently, a complete protein denaturation was performed prior to proteomic analysis for better accessibility of trypsin. After the complete denaturation of the proteins with differential conformation, they are expected to have identical availability for trypsin and the trypsinolysis products of these denatured proteins would be identical, therefore not revealing any information regarding differences in protein conformation. This hypothesis explains why proteomic studies have never reported any tumor-specific changes in blood serum proteins.

Tryptic cleavage under soft conditions is challenging; therefore, to obtain a sufficient amount of peptide ions, it is required to use 'semitrypsin' analysis $(11,12)$. This improves the quality and reliability of the identification of proteins and allows for the identification of the protein structure, including various post-translational modifications.

The aim of the present study was to examine the hypothesis that 1) tumor-specific activity of proteins may be associated with changes in their native conformation, e.g., due to changes in their glycosylation and 2) it is possible to determine changes in the native conformation via the proteolysis products of the protein under soft conditions. Semi-tryptic peptide ions were examined and the biological activity of albumin and inter-alpha-trypsin inhibitor heavy chain 4 (ITIH4) in tumor-free $\mathrm{C} 57 \mathrm{Bl} / 6$ mice and those with B16 melanoma were assessed. The ITIH4 protein is a member of the serpin superfamily (13).

\section{Materials and methods}

Tumor and animal experimentation. B16 melanoma cells were obtained from the bank of tumor strains of the N. N. Blokhin Russian Cancer Research Center (Moscow, Russia). Melanoma cells were transplanted subcutaneously to obtain solid tumors into the right hind limb of C57Bl/6 mice $\left(1 \times 10^{6}\right.$ cells/mouse diluted in $200 \mu 1$ RPMI-1640 medium; PanEcho, Moscow, Russia). The experiments were performed using a total of 250 2- to 3-month-old male $\mathrm{C} 57 \mathrm{Bl} / 6$ and $\mathrm{F} 1$ (C57Bl/6xCBA/Lac) mice (22-24 g) obtained from Stolbovaya Company (Moscow, Russia). The mice received a standard laboratory diet and tap water ad libitum and were kept under a natural light/dark cycle. All experiments were performed in accordance with the National Institutes of Health guidelines (14), the legal regulations for animal experimentation in Russia, and the study was approved by the ethics commiteeb of the Institute of Experimental Diagnosis and Therapy of Tumors of the N. N. Blokhin Russian Cancer Research Center (Moscow, Russia). Blood was extracted according to the protocol described by Fisher et al (5).

Inductive effect of serum protein on tumor growth. To determine the effect of blood serum proteins, fractions of serum proteins were administered intraperitoneally to healthy mice (100 $\mu \mathrm{g} /$ mouse in $400 \mu \mathrm{l} 0.9 \% \mathrm{NaCl}$ solution; PanEcho) two weeks prior to tumor transplantation. Each group included 10 animals.

Serum proteins. Blood serum proteins were collected from normal and from tumor-bearing mice. Serum from B16 tumor-bearing mice was collected 30 days after tumor cell injection. Serum proteins were separated into fractions using ultrafiltration membranes (Millipore, Billerica, MA, USA) under air pressure with a nominal molecular weight limit of $300 \mathrm{kDa}$ [PBMK04310; nominal molecular weight limit (NMWL), 300,000] (fraction 1), 100 kDa (PBHK; NMWL, 100,000 ) (fraction 2) or $50 \mathrm{kDa}(\mathrm{PBQK}$; NMWL, 50,000) (fraction 3). Fraction-3 proteins were diluted in $0.01 \mathrm{M}$ Tris buffer (pH 7.4, containing $0.01 \%$ sodium azide) and transferred onto PD-10 columns (Amersham Biosciences, GE Healthcare, Little Chalfont, UK) (4). Proteins were applied to Sepharose Q FF and Sepharose Blue FF columns (XK 16/20; GE Healthcare). Non-bound serum proteins were eluted with $0.01 \mathrm{M}$ Tris buffer (pH 7.4, containing $0.01 \%$ sodium azide; Sigma-Aldrich, St Louis, MO, USA), while the bound proteins were eluted using a sodium chloride gradient $(0.5 \mathrm{M}, 0.01 \mathrm{M}$ Tris buffer, $\mathrm{pH} 7.4$, with $0.01 \%$ sodium azide; Sigma-Aldrich) (10) with the help of a GP-250 programmed gradient pump (Pharmacia Biotech, GE Healthcare). Protein elution was monitored in a flow cell (SN 20257; $2 \mathrm{~mm}$; GE Healthcare) at $\lambda=280 \mathrm{~nm}$. The samples containing albumin and serpins were collected and analyzed using an HP 8452A diode array spectrophotometer (Agilent Technologies, Santa Clara, CA, USA) measuring the absorption value at $\lambda=280 \mathrm{~nm}$. Subsequently, proteins were intraperitoneally injected at $100 \mu \mathrm{g} /$ mouse in $400 \mu 10.9 \% \mathrm{NaCl}$ solution into healthy mice. After 14 days, $1 \times 10^{6}$ melanoma B16 tumor cells were transplanted into these mice subcutaneously. Each group included 10 animals.

Protein gel electrophoresis. Serpin samples were separated by $12.5 \%$ SDS-PAGE and stained with Coomassie blue (Sigma-Aldrich) according to Laemmli (15). Samples subjected to SDS-PAGE were solubilized in a sample buffer (Sigma-Aldrich) containing $63 \mathrm{mM}$ Tris/ $\mathrm{HCl}, \mathrm{pH} \mathrm{6.8,10 \%}$ (v/v) glycerol, 2\% (w/v) SDS and $30 \mathrm{M}$ bromophenol blue (Sigma-Aldrich). 2-Mercaptoethanol 5\% (v/v) (Sigma-Aldrich) was conditionally added or omitted in the sample buffer. $12.5 \%$ acrylamide gels with a bisacrylamide/acrylamide ratio of 0.8:30 were used. Samples were applied in quantities of 10 and $50 \mu \mathrm{g}$ protein/lane to evaluate all components of the protein complexes. Protein gel electrophoresis was used for determination of the mass of ITIH4 with tumor specific activity.

Assessment of albumin binding using electron spin resonance (ESR). ESR spectra were measured for each sample using a commercially available ESR spectrometer (AXM-09; ESR-Analyzer/MMS; MedInnovation GmbH, Berlin, Germany). The principle of this technique is the measurement of albumin binding variables, achieved by a fatty acid spin probe. The binding variables of the spin probe were determined at different permutations of the ethanol concentration as well as the ratio of spin probe and albumin concentration. Variation of the ethanol concentration allowed for the assessment of binding variables of the spin probe to albumin under different hydrophobic condi- 
Table I. Semi-tryptic peptides of albumin identified in tumor-bearing mice and tumor-free mice.

\begin{tabular}{lcc}
\hline $\begin{array}{l}\text { B16 melanoma } \\
\text { (m/z of peptide ion) }\end{array}$ & $\begin{array}{c}\text { Tumor-free mice } \\
\text { (m/z of peptide ion) }\end{array}$ & $\begin{array}{c}\text { Amino acids } \\
\text { (start-end) }\end{array}$ \\
\hline 777.3831 & 777.4126 & $199-205$ \\
1149.6602 & 1149.6265 & $66-75$ \\
1250.6354 & 1250.5844 & $35-44$ \\
1439.8602 & 1439.7895 & $439-452$ \\
1479.9253 & 1479.8529 & $45-57$ \\
1681.9364 & 1681.8677 & $243-257$ \\
3870.3227 & 3869.8416 & $25-57$
\end{tabular}

Table II. Semi-tryptic peptides of albumin identified in tumor-bearing mice only.

\begin{tabular}{lc}
\hline $\begin{array}{l}\text { Peptide ion } \\
(\mathrm{m} / \mathrm{z})\end{array}$ & $\begin{array}{c}\text { Amino acids } \\
\text { (start-end) }\end{array}$ \\
\hline 960.4622 & $484-490$ \\
1019.6167 & $234-242$ \\
1272.6371 & $211-221$ \\
1902.0049 & $153-168$ \\
2711.6178 & $35-57$ \\
2982.4342 & $563-588$ \\
3369.7339 & $559-588$ \\
342.693 & $410-438$ \\
\hline
\end{tabular}

tions. Changes in the ratio of spin probe to albumin enabled the measurement of the binding affinity of albumin to the spin probe.

Commercial 16-doxyl stearic acid (Sigma-Aldrich) was used as spin probe. This compound was selected due to the exceptionally high binding constant of albumin to stearic acid $\left(6.9 \times 10^{7}\right.$ $1 / \mathrm{mol}$ ), which produces $>99.9 \%$ binding of the spin probe to albumin. Ethanol, extra pure, (Merck Millipore) was used for modifying the binding affinity of the fatty acid spin probe to albumin. The final concentrations of ethanol (mol/l) and spin probe $\left(10^{-3} \mathrm{~mol} / \mathrm{l}\right)$ were 2.9 and 0.83 in aliquot $1,3.4$ and 1.61 in aliquot 2 , and 3.8 and 2.34 in aliquot 3 , respectively (16).

Mass spectrometry. For mass spectrometric analysis, samples of protein were only used after purification using Sephadex Q FF columns. Proteins were diluted in $50 \mathrm{mM}$ ammonium bicarbonate solution and transferred onto PD-10 columns (W359685; GE Healthcare). The final concentration of the proteins in the samples was $1 \mathrm{mg} / \mathrm{ml}$. Solely following column chromatography without electrophoresis, the samples were incubated at $37^{\circ} \mathrm{C}$ in $100 \mu 120 \mathrm{mM}$ ammonium bicarbonate containing $5 \mathrm{ng} / \mu \mathrm{l}$ trypsin, chymotrypsin and $10 \%$ acetonitrile overnight (all from Sigma-Aldrich). Peptides were concentrated using SEP-PAK C18 cartridges (Millipore).

Mass spectra were recorded using an Ultraflex Extreme MALDI-TOF/TOF mass spectrometer (Bruker Daltonics, Billerica, MA, USA) equipped with a neodymium laser. To analyze mass spectra, FlexAnalysis software, version 3.3
(Bruker Daltonics, Bremen, Germany) was used. Aliquots of the samples were mixed on a steel target with a solution of 2,5-dihydroxybenzoic acid $(20 \mathrm{mg} / \mathrm{ml})$ in $30 \%(\mathrm{v} / \mathrm{v})$ acetonitrile (Sigma-Aldrich) with $0.5 \%(\mathrm{v} / \mathrm{v})$ trifluoroacetic acid. The $[\mathrm{MH}]^{+}$ molecular ions were analyzed in linear (proteins) or reflector (peptides) mode, and the $\mathrm{m} / \mathrm{z}$ ratios were accurate to $30 \mathrm{ppm}$. Fragment ion spectra were obtained in Lift mode with a mass accuracy of better than $1 \mathrm{Da}$. Proteases cleaved peptides were used in the peptide mixtures. The data were processed using Flex Analysis 2.2 software (Bruker Daltonics), and peaks of trypsin fragments contained in samples were used for calibration. The following search parameters were used: Accuracy of mass determination $=100 \mathrm{ppm}$, NCBInr database, Rodentia taxon (rodent), one missed cleavage and possible methionine oxidation. The proteins were identified using the Mascot version 2.2.07 search software (peptide fingerprint option; www.matrixscience.com). The search was conducted in the NCBI databases and/or EST vertebrates. For the search of candidate proteins in combined ms + (ms-ms) data, Biotools, version 3.0 (Bruker Daltonics) was used. If the value of the Score parameter calculated for each protein exceeded 70 , identification was accepted as reliable.

Statistical analysis. Statistical evaluation was performed using Fisher's exact test or Student's t-test. Data are presented as the mean \pm standard deviation and Microsoft Office Excel (14.0.6112.5000) software was used for statistical analysis. $\mathrm{P}<0.05$ was considered to indicate a statistically significant difference between values.

\section{Results}

Tumor-free mice and mice with B16 melanoma have differential proteolytic fragments of serum albumin. By means of column chromatography, a fraction corresponding to albumin was extracted from the blood serum of tumor-free mice and the blood serum of mice with B16 melanoma. Subsequent proteomic analysis identified the serum precursor of albumin in the two cases (Fig. 1A and B). Thus, albumin extracted from mice with the tumor has the same amino acid sequence as albumin extracted from the tumor-free mice.

In addition, the data presented in Fig. 1A and B show that proteolytic analysis of the albumin from tumor-free mice identified a greater number of semi-tryptic peptide ions compared with that in mice with B16 melanoma (64 vs 15 peptides, respectively). Normal mice and those with B16 melanoma had seven peptides 
A

\begin{tabular}{|c|c|c|c|c|c|c|c|c|}
\hline & & $18 \mathrm{t}$ & ( & & & & & \\
\hline Observed & $\operatorname{Mr}$ (expt) & $\operatorname{Mr}(\mathrm{calc})$ & & Start & & & Ions & Peptide \\
\hline $\begin{array}{l}761.3758 \\
774.3856\end{array}$ & $\begin{array}{l}760.3685 \\
773.3783\end{array}$ & $\begin{array}{l}760.3967 \\
773.4072\end{array}$ & $\begin{array}{l}-36.97 \\
-37.31\end{array}$ & $\begin{array}{r}563 \\
89\end{array}$ & $\begin{array}{l}-569 \\
-95\end{array}$ & $\begin{array}{l}0 \\
0\end{array}$ & -- & $\begin{array}{l}\text { K. ATAEQLR.T + Deamidated (NQ) } \\
\text { K.SLHTLFG.D }\end{array}$ \\
\hline 777.4126 & 776.4053 & 776.3738 & 40.6 & & -205 & 0 & -- & $\begin{array}{l}\text { K.SLATLFG.D } \\
\text { K.ESCLTPK.L }\end{array}$ \\
\hline 808.4006 & 807.3933 & 807.3650 & 35.0 & & & 0 & -- & R.LSQTPPN.A + 2 Deamidated (NQ) \\
\hline 828.4451 & 827.4379 & 827.4752 & -45.16 & & -413 & 1 & --- & E.EPKNLVK.T + Deamidated (NQ) \\
\hline $\begin{array}{l}830.3876 \\
873.4370\end{array}$ & $\begin{array}{l}829.3803 \\
872.4297\end{array}$ & $\begin{array}{l}829.3970 \\
872.4141\end{array}$ & $\begin{array}{r}-20.10 \\
17.9\end{array}$ & $\begin{array}{r}355 \\
361\end{array}$ & $\begin{array}{l}-360 \\
-367\end{array}$ & $i$ & --- & $\begin{array}{l}\text { F.LYYYSR.R } \\
\text { R.RPDYSV,S }\end{array}$ \\
\hline 894.4789 & 893.4716 & $\begin{array}{l}872.4141 \\
893.4494\end{array}$ & $\begin{array}{l}17.9 \\
24.9\end{array}$ & 250 & $\begin{array}{l}=367 \\
-257\end{array}$ & ${ }_{0}^{1}$ & $=$ & $\begin{array}{l}\text { R.RHPDYSV.S } \\
\text { N.ADFAEITK.L }\end{array}$ \\
\hline 918.4799 & 917.4726 & 917.4528 & 21.6 & 536 & -543 & 0 & --- & S.DICTLPEK.E \\
\hline 940.4717 & 939.4644 & 939.4888 & -25.99 & & & 1 & --- & -.MKWVTFL.L + Oxidation (M) \\
\hline 962.5168 & 961.5095 & 961.5233 & $-14 \cdot 28$ & & -434 & 0 & --- & G.FQNAILVR. $Y+2$ Deamidated (NQ) \\
\hline 984.4970 & $\begin{array}{l}983.4897 \\
988.4747\end{array}$ & $\begin{array}{r}983.5076 \\
988.4726\end{array}$ & -18.24 & & & 0 & $=--$ & 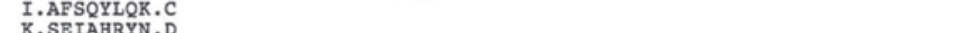 \\
\hline $\begin{array}{r}989.4819 \\
1019.6093\end{array}$ & $\begin{array}{r}988.4747 \\
1018.6021\end{array}$ & $\begin{array}{r}988.4726 \\
1018.6215\end{array}$ & & 29 & $\begin{array}{r}36 \\
9\end{array}$ & $\frac{1}{1}$ & $=--$ & $\begin{array}{l}\text { K.SEIAHRYN.D } \\
\text { M. KWYTFLLL, }\end{array}$ \\
\hline 1038.4667 & 1037.4594 & $\begin{array}{l}1037.4708 \\
1037\end{array}$ & -19.99 & 302 & $=309$ & 0 & $=$ & T.CCDKPLLK. $\mathrm{K}+$ Cysteiny 1 \\
\hline 1062.4408 & 1061.4335 & 1061.4014 & 30.3 & 222 & -229 & 1 & --- & R.MKCSSMQR.F + Deamidated (NQ); Cysteinyl (C) \\
\hline 1132.5209 & 1131.5136 & 1131.4576 & 49.5 & 143 & -152 & 0 & --- & P.EAEAMCTSFK.E + Oxidation (M) \\
\hline $\begin{array}{l}1149.6265 \\
1179.5957\end{array}$ & $\begin{array}{l}1148.6192 \\
1178.5884\end{array}$ & & 9.96 & $\begin{array}{r}66 \\
537\end{array}$ & $=75$ & 0 & $\overline{---}$ & K.LVQEVTDFAK.T \\
\hline $\begin{array}{l}1179.5957 \\
1250.5844\end{array}$ & $\begin{array}{l}1178.5884 \\
1249.5771\end{array}$ & $\begin{array}{l}1178.5675 \\
1249.5727\end{array}$ & $\begin{array}{l}17.8 \\
3.52\end{array}$ & $\begin{array}{r}537 \\
35\end{array}$ & $=545$ & ${ }_{0}^{1}$ & $=$ & $\begin{array}{l}\text { D.CTCLPEKEK.Q + Cysteiny1 (c) } \\
\text { R.YNDLGEOHFK.G }\end{array}$ \\
\hline 1282.5380 & 1281.5307 & 1281.5547 & -18.74 & 331 & $=341$ & 0 & 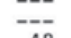 & A.ADFVEDQEVCK, N \\
\hline 1292.6641 & 1291.6568 & 1291.6349 & 16.9 & 169 & -178 & 1 & 48 & R.RHPYFYAPEL.L \\
\hline $\begin{array}{l}1346.5172 \\
1406.7102\end{array}$ & $\begin{array}{l}1345.5100 \\
1405.7029\end{array}$ & $\begin{array}{l}1345.5166 \\
1405\end{array}$ & -4.92 & 106 & -117 & 0 & --- & $\begin{array}{l}\text { R.ENYGELADCCTK.Q + Deamidated (NQ) } \\
\text { R }\end{array}$ \\
\hline $\begin{array}{l}1406.7102 \\
1439.7895\end{array}$ & $\begin{array}{l}1405.7029 \\
1438.7823\end{array}$ & $\begin{array}{l}1405.6738 \\
1438.7780\end{array}$ & $\begin{array}{l}20.7 \\
2.98\end{array}$ & $\begin{array}{r}34 \\
439\end{array}$ & $\begin{array}{l}-44 \\
-452\end{array}$ & $\frac{1}{0}$ & --- & $\begin{array}{l}\text { H.RYNDLGEQHF.G } \\
\text { K.APQVSTPTVEAAR.N }\end{array}$ \\
\hline 1479.8529 & 1478.8456 & 1478.8497 & $\begin{array}{l}-2.74 \\
-2.74\end{array}$ & & $=57$ & 0 & -- & 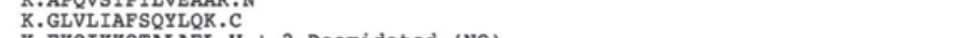 \\
\hline 1501.8569 & 1500.8497 & 1500.8399 & 6.54 & 544 & -556 & 3 & -- & K.EKQIKRQTALAEL.V + 2 Deamidated (NQ) \\
\hline $\begin{array}{l}1530.6545 \\
1552.6675\end{array}$ & $\begin{array}{l}1529.6472 \\
1551.6602\end{array}$ & 1529.6569 & -6.29 & 118 & -129 & 1 & --- & K.QEPERNECFLQH.K + Deamidated (NQ) \\
\hline $\begin{array}{l}1552.6675 \\
1626.7882\end{array}$ & $\begin{array}{l}1551.6602 \\
1625.7809\end{array}$ & $\begin{array}{l}1551.6044 \\
1625.7243\end{array}$ & $\begin{array}{l}36.0 \\
34.8\end{array}-1-100$ & $\begin{array}{r}572 \\
334\end{array}$ & $\begin{array}{l}-584 \\
-347\end{array}$ & $\stackrel{0}{1}$ & $=$ & 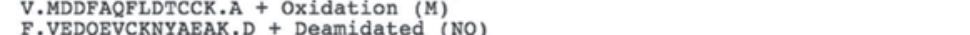 \\
\hline $\begin{array}{l}1626.7882 \\
1681.8677\end{array}$ & $\begin{array}{l}1625.7809 \\
1680.8605\end{array}$ & $\begin{array}{l}1625.7243 \\
1680.8359\end{array}$ & $\begin{array}{l}34.8 \\
14.6\end{array}$ & $\begin{array}{l}334 \\
243\end{array}$ & $\begin{array}{l}-347 \\
-257\end{array}$ & ${ }_{0}^{1}$ & $\overline{---}$ & $\begin{array}{l}\text { R.LSQTFPNADFAEITK.L } \\
\text { R. Deamidated (NQ) }\end{array}$ \\
\hline 1703.8686 & 1702.8613 & 1702.8566 & 2.78 & 425 & -438 & 1 & -- & E. YGFQNAILVRYTQK.A + 3 Deamidated (NQ) \\
\hline $\begin{array}{l}1725.8446 \\
1840.8889\end{array}$ & $\begin{array}{l}1724.8373 \\
1839\end{array}$ & 1724.8342 & $\begin{array}{r}1.81 \\
-16,33\end{array}$ & 24 & $=37$ & 3 & $=--$ & \\
\hline $\begin{array}{l}1840.8889 \\
1888.8641\end{array}$ & $\begin{array}{l}1839.8817 \\
1887.8568\end{array}$ & $\begin{array}{l}1839.91117 \\
1887.8593\end{array}$ & $\begin{array}{r}-16 \cdot 33 \\
-1.34\end{array}$ & $\begin{array}{l}211 \\
282\end{array}$ & $\begin{array}{l}-225 \\
-298\end{array}$ & 4 & --- & K. BRALVSSVRQRMKCS.S + Cysteiny 1 (C) \\
\hline $\begin{array}{l}1888.8641 \\
1901.9604\end{array}$ & $\begin{array}{l}1887.8568 \\
1900.9531\end{array}$ & $\begin{array}{l}1887.8593 \\
1900.9570\end{array}$ & $\begin{array}{l}-1.34 \\
-2.05\end{array}$ & $\begin{array}{l}282 \\
419\end{array}$ & $\begin{array}{l}-2988 \\
-434\end{array}$ & ${ }_{1}^{1}$ & $=$ & 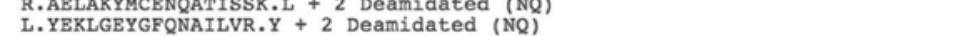 \\
\hline 1918.8436 & 1917.8363 & 1917.8567 & -10.60 & 50 & -65 & 1 & -- & I.AFSQYLQKCSYDEHAK.L + Deamidated (NQ) \\
\hline 1973.9300 & 1972.9227 & 1972.9598 & -18.80 & 258 & -275 & 2 & -- & 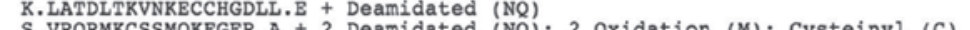 \\
\hline $\begin{array}{l}2123.9409 \\
2267.1002\end{array}$ & 2122.9336 & 2122.9380 & -2.06 & 218 & $=-233$ & 4 & $-\overline{-}$ & 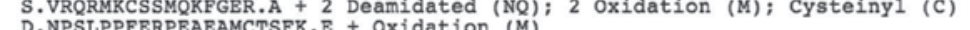 \\
\hline $\begin{array}{l}2267.1002 \\
2285.0967\end{array}$ & $\begin{array}{l}2266.0929 \\
2284.0894\end{array}$ & $\begin{array}{l}2266.0398 \\
2284.0827\end{array}$ & $\begin{array}{l}23.94 \\
2.93\end{array}$ & $\begin{array}{l}133 \\
584\end{array}$ & $\begin{array}{l}-152 \\
-604\end{array}$ & ${ }_{3}^{0}$ & $=$ & $\begin{array}{l}\text { D.NPSLPPFERPEARAMCTSFK.E + oxidation (M) } \\
\text { C.KAADKDTCFSTEPNLVTRCK.D + Deamidated (NQ) }\end{array}$ \\
\hline 2302.0979 & $\begin{array}{l}2284.0894 \\
2301.0907\end{array}$ & 2301.1351 & -19.31 & 389 & -409 & 0 & --- & 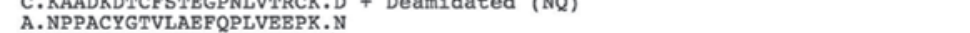 \\
\hline 2383.1272 & 2382.1200 & 2382.1063 & & & -14 & 1 & -- & R.NECFLQHKDDNPSLPPI \\
\hline 2767.3422 & 2766.3349 & 2766.3278 & 2.57 & & & 2 & --- & R.VGTKCCTLPEDQRLPCVEDYLSAIL: \\
\hline 2829.3899 & 2828.3826 & 2828.2601 & 43.3 & 453 & 6 & 3 & & R.NLGRVGTKCCTLPEDQRLPCVEDY.L \\
\hline 2846.3625 & 2845.35 & 2845.3997 & -15.63 & 445 & 9 & 3 & & 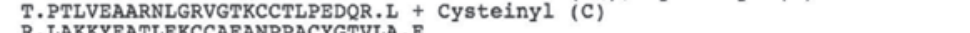 \\
\hline .4300 & 2856.42 & 2856.38 & 12.9 & 373 & -39 & 3 & -- & R.LAKKYEA \\
\hline & 2946.4 & 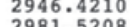 & 0.09 & 284 & -309 & 2 & -- & E.LAKYMCENQATISSKLQTCCDKPLLR.K +2 Deamidated (NQ \\
\hline $\begin{array}{l}2982.5004 \\
3086.5296\end{array}$ & $\begin{array}{l}2981.4931 \\
3085.5223\end{array}$ & 2981. & $\begin{array}{r}-9.28 \\
22.6\end{array}$ & $\begin{array}{r}45 \\
414\end{array}$ & & 2 & $-\bar{c}$ r & K. GLVLAFSQYLQKCSYDEHAKLVQEV T + Deamidated (NQ) \\
\hline $\begin{array}{l}3086.5296 \\
3110.6117\end{array}$ & $\begin{array}{l}3085.5223 \\
3109.6044\end{array}$ & $\begin{array}{l}3085.4525 \\
3109.5471\end{array}$ & $\begin{array}{l}22.6 \\
18.4\end{array}$ & $\begin{array}{l}414 \\
435\end{array}$ & $\begin{array}{l}-438 \\
-462\end{array}$ & $\frac{2}{4}$ & --- & R.YTOKAPOVSTPTLVEAARNLGRVGTKCC.T + Cysteiny 1 (C) \\
\hline 3232.5635 & 3231.5563 & 3231.5614 & -1.58 & 560 & -588 & 3 & -- & H.KPKATAEQLKTVMDDFAQFLDTCCKAADK.D + Oxidation (M) \\
\hline & 3240.6064 & 3240.6231 & -5.14 & 440 & -469 & 3 & -- & A.PQVSTPTLVEAA \\
\hline 5743 & 3248.5670 & 3248.4233 & 44.3 & 183 & -210 & 2 & 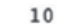 & A.EQYNEILTOCCAEADKESCLTPRLDGVK. E +2 \\
\hline 5911 & 3266.58 & 3266.53 & 15.6 & 414 & -441 & 3 & & K.TNCDLYEKLG \\
\hline & 336 & 3368.63 & 9.1300100 & & -6 & 2 & & EHAR.L +2 Deam \\
\hline & 341 & 34. & -10.5 & 281 & $-3 c$ & 3 & - & D.RAELAKYMCENQATISSKLQTCCDKPLLK $K \mathrm{~K}$ + oxidation \\
\hline 343 & 3437.53 & $\begin{array}{ll}3437 \\
2557\end{array}$ & 16.5 & 461 & -48 & 2 & & X.CCTLPEDQRLPCVEDYLSAILNRVCLL. $\mathrm{H}+2$ Deamidated \\
\hline & $\begin{array}{l}3551.56 \\
3868.83\end{array}-10-100$ & $\begin{array}{l}3551.5135 \\
3869.0012\end{array}$ & $-43 . i^{3}$ & 94 & $=-122$ & 3 & & 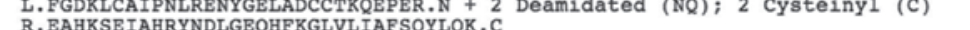 \\
\hline & 3984.86 & 3984.8617 & -0.74 & & & 2 & & R. LLKKAHCLSEVEHDTMPADLPAIAADFVEDQEVCK. $\mathrm{N}+$ Deamida \\
\hline 4082.41 & 408 & 4081.5726 & -40 . & 106 & -13 & 3 & --- & Cysteiny1 (C) \\
\hline 4204.5986 & 4203.5913 & 4203.7377 & -34.82 & 106 & 139 & 3 & -- & FNYCRIADCCTE \\
\hline 6344.6375 & 6343.6302 & 6343.6570 & -4.23 & 106 & -160 & 4 & --- & $\begin{array}{l}\text { R.ENYGELADCCTKQPERNECFLQHDDNPSLPPFERPEAEAMCTSFKENPTFMG.H } \\
+5 \text { Deamidated (NQ); Oxidation (M) }\end{array}$ \\
\hline
\end{tabular}

B

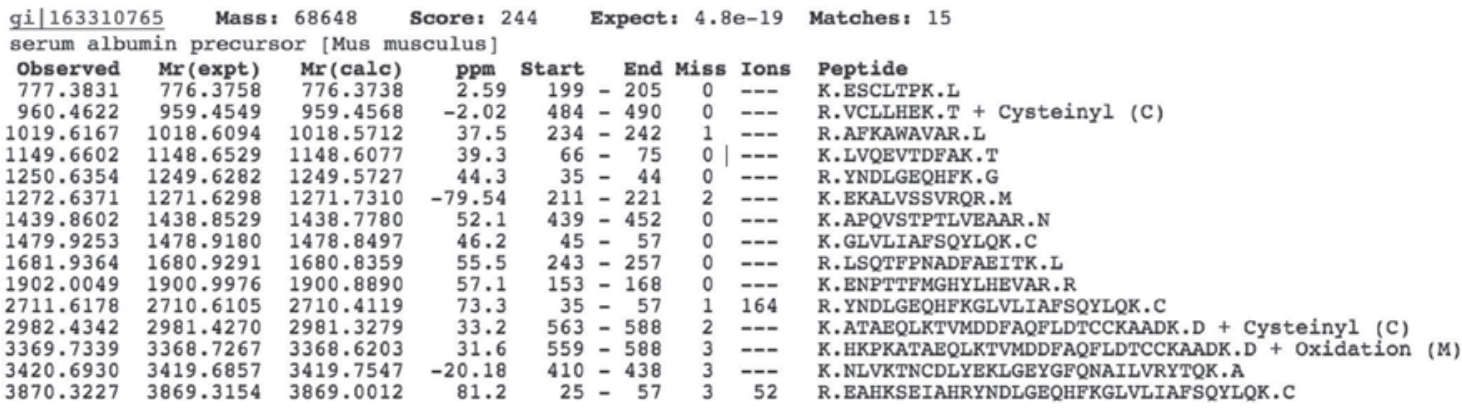

Figure 1. Summary report of the identification of serum albumin precursor and semi-tryptic peptides (A) for tumor-free mice and (B) for mice with B16 melanoma. $\mathrm{N}$, arginine; $\mathrm{Q}$, glutamine; $\mathrm{C}$, cysteine; $\mathrm{M}$, methionine.

in common, and eight peptides were found exclusively in mice with B16 melanoma (Tables I and II). However, a number of the peptides which identified in mice with B16 melanoma partially or completely overlapped with a significant number of the peptides identified in tumor-free mice. This resulted in a larger number of identified peptides in tumor-free mice compared with that in mice with B16 melanoma (Table III).

In tumor-free mice, seven peptides were identified to partially overlap with those identified in mice with B16 mela- noma with regard to their amino acid sequences at 35-57 and the $\mathrm{m} / \mathrm{z}$ ratio of $2,711.6178$, and two peptides were identified to partially overlap with regard to their amino acid sequences at $211-221$ and the $\mathrm{m} / \mathrm{z}$ ratio of $1,272.6371$. Furthermore, in tumor-free mice, two peptides were identified to partially overlap with those found in mice with B16 melanoma with regard to their amino acid sequences at 243-257 and the m/z ratio of $1,681.9364$. In tumor-free mice, six peptides were identified to partially or completely overlap with those from 
Table III. Overlapping peptides of albumin in tumor-bearing mice and in tumor-free mice.

\begin{tabular}{|c|c|c|c|}
\hline Animals & $\begin{array}{l}\text { Peptide ion } \\
\qquad(\mathrm{m} / \mathrm{z})\end{array}$ & Start-end & Amino acid sequence \\
\hline B16 melanoma & 2711.6178 & $35-57$ & R.YNDLGEQHFKGLVLIAFSQYLQK.C \\
\hline Tumor-free mice & 1725.8446 & $24-37$ & R.REAHKSEIAHRYND.L \\
\hline Tumor-free mice & 989.4819 & $29-36$ & K.SEIAHRYN.D \\
\hline Tumor-free mice & 1406.7102 & $34-44$ & H.RYNDLGEQHFK.G \\
\hline Tumor-free mice & 3369.6769 & $37-65$ & $\begin{array}{l}\text { N.DLGEQHFKGLVLIAFSQYLQKCSYDEHAK.L } \\
+2 \text { deamidated (NQ) }\end{array}$ \\
\hline Tumor-free mice & 2982.5004 & $45-70$ & $\begin{array}{l}\text { K.GLVLIAFSQYLQKCSYDEHAKLVQEV.T } \\
\text { + deamidated (NQ) }\end{array}$ \\
\hline Tumor-free mice & 984.497 & $50-57$ & I.AFSQYLQK.C \\
\hline Tumor-free mice & 1918.8436 & $50-65$ & I.AFSQYLQKCSYDEHAK.L + deamidated (NQ) \\
\hline B16 melanoma & 1272.6371 & $211-221$ & K.EKALVSSVRQR.M \\
\hline Tumor-free mice & 1840.8889 & $211-225$ & K.EKALVSSVRQRMKCS.S + cysteinyl (C) \\
\hline Tumor-free mice & 2123.9409 & $218-233$ & S.VRQRMKCSSMQKFGER.A + 2 deamidated (NQ) \\
\hline Tumor-free mice & 1681.8677 & $243-257$ & R.LSQTFPNADFAEITK.L \\
\hline B16 melanoma & 1681.9364 & $243-257$ & R.LSQTFPNADFAEITK.L \\
\hline Tumor-free mice & 808.4006 & $243-249$ & R.LSQTFPN.A + 2 deamidated (NQ) \\
\hline Tumor-free mice & 894.4789 & $250-257$ & N.ADFAEITK.L \\
\hline B16 melanoma & 3420.693 & $410-438$ & K.NLVKTNCDLYEKLGEYGFQNAILVRYTQK.A \\
\hline Tumor-free mice & 3086.5296 & $414-438$ & $\begin{array}{l}\text { K.TNCDLYEKLGEYGFQNAILVRYTQK.A } \\
\text { + Deamidated (NQ) }\end{array}$ \\
\hline Tumor-free mice & 3267.5911 & $414-441$ & $\begin{array}{l}\text { K.TNCDLYEKLGEYGFQNAILVRYTQKAPQ.V } \\
+5 \text { deamidated (NQ) }\end{array}$ \\
\hline Tumor-free mice & 1901.9604 & $419-434$ & L.YEKLGEYGFQNAILVR.Y + 2 deamidated (NQ) \\
\hline Tumor-free mice & 1703.8686 & $425-438$ & E.YGFQNAILVRYTQK.A + 3 deamidated (NQ) \\
\hline Tumor-free mice & 962.5168 & $427-434$ & G.FQNAILVR.Y + 2 deamidated (NQ) \\
\hline Tumor-free mice & 3110.6117 & $435-462$ & $\begin{array}{l}\text { R.YTQKAPQVSTPTLVEAARNLGRVGTKCC.T } \\
+ \text { cysteinyl }(\mathrm{C})\end{array}$ \\
\hline Tumor-free mice & 1439.7895 & $439-452$ & K.APQVSTPTLVEAAR.N \\
\hline B16 melanoma & 1439.8602 & $439-452$ & K.APQVSTPTLVEAAR.N \\
\hline Tumor-free mice & 3241.6137 & $440-469$ & $\begin{array}{l}\text { A.PQVSTPTLVEAARNLGRVGTKCCTLPEDQR.L } \\
+2 \text { deamidated (NQ) }\end{array}$ \\
\hline Tumor-free mice & 2846.3625 & $445-469$ & T.PTLVEAARNLGRVGTKCCTLPEDQR.L + cysteinyl (C) \\
\hline B16 melanoma & 3369.7339 & $559-588$ & $\begin{array}{l}\text { K.HKPKATAEQLKTVMDDFAQFLDTCCKAADK. } \\
\text { D + oxidation }(\mathrm{M})\end{array}$ \\
\hline B16 melanoma & 2982.4342 & $563-588$ & $\begin{array}{l}\text { K.ATAEQLKTVMDDFAQFLDTCCKAADK. } \\
\text { D + cysteinyl (C) }\end{array}$ \\
\hline Tumor-free mice & 3232.5635 & $560-588$ & $\begin{array}{l}\text { H.KPKATAEQLKTVMDDFAQFLDTCCKAADK. } \\
\text { D + oxidation (M) }\end{array}$ \\
\hline Tumor-free mice & 761.3758 & $563-569$ & K.ATAEQLK.T + deamidated (NQ) \\
\hline Tumor-free mice & 1552.6675 & $572-584$ & V.MDDFAQFLDTCCK.A + oxidation (M) \\
\hline Tumor-free mice & 2285.0967 & $584-604$ & C.KAADKDTCFSTEGPNLVTRCK.D + deamidated (NQ) \\
\hline
\end{tabular}

$\mathrm{N}$, arginine; $\mathrm{Q}$, glutamine; $\mathrm{C}$, cysteine; $\mathrm{M}$, methionine.

mice with B16 melanoma in their amino acid sequences at 410-438 and an m/z 3,420.693. In addition, in tumor-free mice, two peptides were identified to partially overlap with peptides from mice with B16 melanoma in their amino acid sequences at 439-452 and the $\mathrm{m} / \mathrm{z}$ ratios of $1,439.8602$ and 1,439.7895. Finally, in tumor-free mice, four peptides were identified to partially or fully overlap with two peptides in mice with B16 melanoma regarding the amino acid sequences 559-588 with the $\mathrm{m} / \mathrm{z}$ ratio of 3,369.7339 and 563-588 with the $\mathrm{m} / \mathrm{z}$ ratio of $2,982.4342$. The large number and variation of proteolysis products of the serum proteins from tumor-free mice compared to those from mice with melanoma B16, indicates the mobility of these specific regions in the former and the rigidity of the same parts of the molecule in the latter. The 


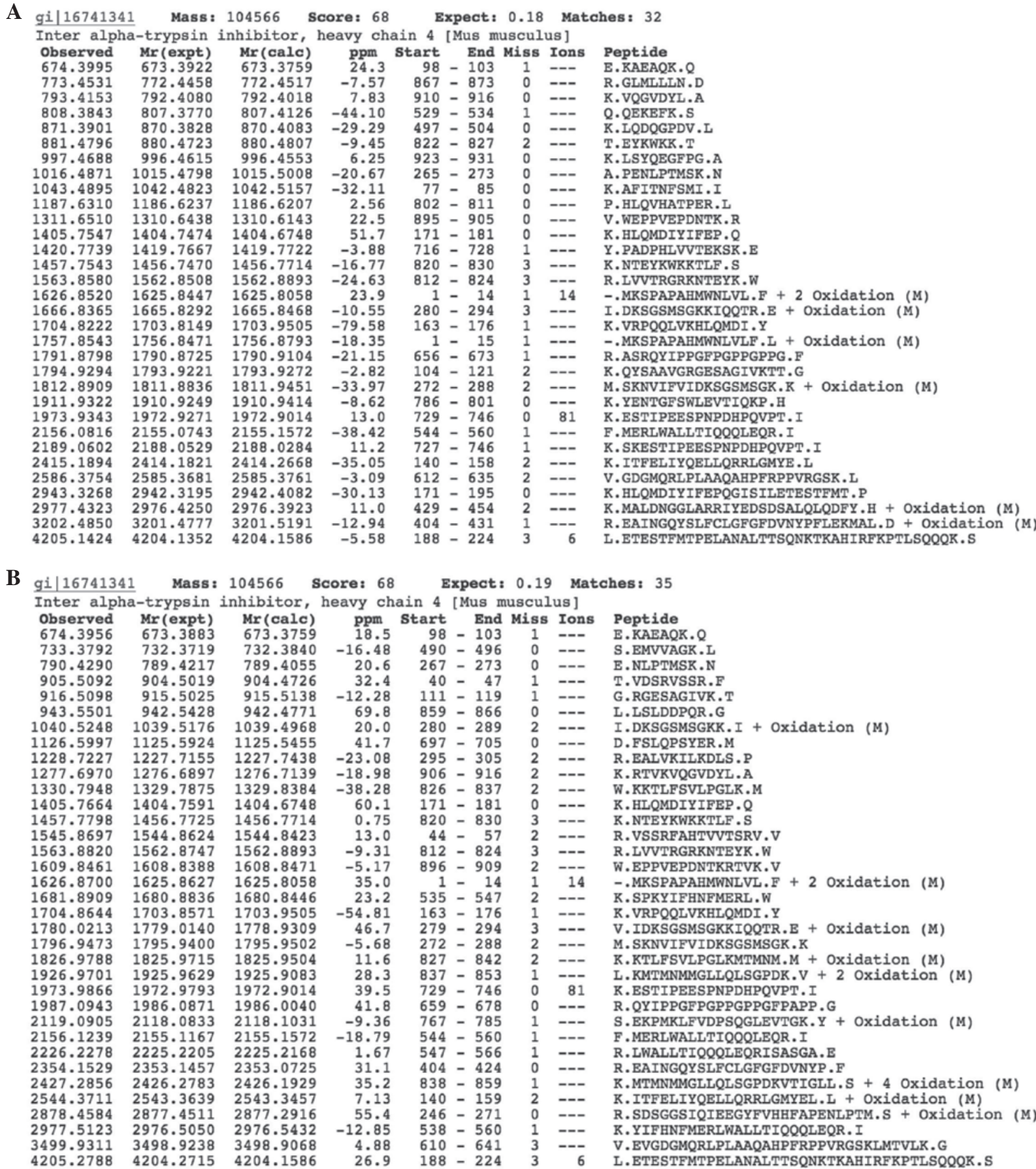

Figure 2. Summary report of the identification of the inter-alpha-trypsin inhibitor heavy chain 4 and semi-tryptic peptides (A) for tumor-free mice and (B) for mice with B16 melanoma. M, methionine.

increase in rigidity of the albumin molecule during tumor growth may be due to the formation of cross-links within the molecule. This process may be similar to the transformation of fibrin to form internal cross-links (17). This process may make proteins in animals with tumors more rigid compared to those of tumor-free animals and leads to a decrease in the number of proteolysis products.

Tumor-free mice and mice with B16 melanoma have differential proteolytic fragments of serum ITIH4. Another protein isolated by column chromatography was the protein fraction corresponding to ITIH4. Similarly to the case of albumin, it was necessary to verify that proteins with the same amino acid sequence were analyzed in the protein extracted from the blood serum of the mice with B16 melanoma and that from the blood serum of tumor-free mice. As shown in Fig. 2A and B, proteomic analysis identified ITIH4 in the two groups.

The total quantity of identified peptides following proteolysis of ITIH4 from the serum of tumor-free mice was 32, that of mice with B16 melanoma was 35 . Thus, by contrast with albumin, in the case of serpin, no significant difference was observed in the number of identified peptide fragments of ITIH4. As discussed above, the lack of variations in the number of identified peptides in plasma during tumor growth may be explained by various 
Table IV. Semi-tryptic peptides of the inter-alpha-trypsin inhibitor heavy chain 4 identified in tumor-bearing and tumor-free mice.

\begin{tabular}{lcc}
\hline $\begin{array}{l}\text { Peptide ion in } \\
\text { B16 melanoma }(\mathrm{m} / \mathrm{z})\end{array}$ & $\begin{array}{c}\text { Peptide ion in } \\
\text { tumor-free mice (m/z) }\end{array}$ & $\begin{array}{c}\text { Amino acids } \\
\text { (start-end) }\end{array}$ \\
\hline 674.3956 & 674.3995 & $98-103$ \\
1405.7664 & 1405.7547 & $171-181$ \\
1457.7798 & 1457.7543 & $820-830$ \\
1563.882 & 1563.858 & $812-824$ \\
1626.8700 & 1626.852 & $1-14$ \\
1704.8644 & 1704.8222 & $163-176$ \\
1973.9866 & 1973.9343 & $729-746$ \\
2156.1239 & 2156.0816 & $544-560$ \\
1796.9473 & 1812.8909 & $272-288$ \\
4205.2788 & 4205.1424 & $188-224$ \\
\hline
\end{tabular}

Table V. Semi-tryptic peptides of the inter-alpha-trypsin inhibitor heavy chain 4 identified in tumor-bearing mice only.

\begin{tabular}{lc}
\hline $\begin{array}{l}\text { Peptide ion } \\
(\mathrm{m} / \mathrm{z})\end{array}$ & $\begin{array}{c}\text { Amino acids } \\
\text { (start to end })\end{array}$ \\
\hline 733.3792 & $490-496$ \\
790.4290 & $267-273$ \\
905.5092 & $40-47$ \\
916.5098 & $111-119$ \\
943.5501 & $859-866$ \\
1040.5248 & $280-289$ \\
1126.5997 & $697-705$ \\
1228.7227 & $295-305$ \\
1277.6970 & $906-916$ \\
1330.7948 & $826-837$ \\
1545.8697 & $44-57$ \\
1609.8461 & $896-909$ \\
1780.0213 & $279-294$ \\
1826.9788 & $827-842$ \\
1926.9701 & $837-853$ \\
1987.0943 & $659-678$ \\
2119.0905 & $767-785$ \\
2354.1529 & $404-424$ \\
2427.2856 & $838-859$ \\
2544.3711 & $140-159$ \\
2977.5123 & $538-560$ \\
3499.9311 & $610-641$ \\
\hline
\end{tabular}

mechanisms of modification of the proteins in the body. Serpin, unlike albumin, is a glycosylated protein and its spatial structure changes due to glycosylation, not due to internal cross-links. Glycosylation-induced changes of blood serum proteins during tumor growth are discussed above (2).

Among the identified peptide fragments of ITIH4 following proteolysis, 10 common peptides were identified among the proteins derived from tumor-bearing mice and those obtained from tumor-free mice (Table IV), while the remaining peptide fragments were unique in the tumor-bearing mice (Table V, $\mathrm{n}=22)$ and the tumor-free mice $(\mathrm{n}=25)$.

Among the peptides identified in tumor-free mice, twelve peptides were detected that partially or completely overlapped with thirteen peptides detected in mice with B16 melanoma (Table VI). A peptide fragment with an amino acid sequence of 104-121 and an m/z of 1,794.9294 was detected in tumor-free mice. This peptide completely overlapped with the peptide fragment with the amino acid sequence 111-119 and an m/z of 916.5098 found in mice with B16 melanoma. Furthermore, a peptide with the amino acid sequence of 140-158 and an m/z ratio of 2,415.1894 detected in tumor-free mice partially overlapped with a peptide with the amino acid sequence $140-159$ and the $\mathrm{m} / \mathrm{z}$ of $2,544.3711$ found in mice with B16 melanoma. The peptide with the amino acid sequence of 171-195 and the $\mathrm{m} / \mathrm{z}$ of 2,943.3268 detected in tumor-free mice completely overlapped with the peptide with the amino acid sequence 171-181 found in mice with B16 melanoma (m/z, 1,405.7664) and in tumor-free mice $(\mathrm{m} / \mathrm{z} 1,405.7547)$. The peptide with the amino acid sequence of 265-273 and the $\mathrm{m} / \mathrm{z}$ of 1,016.4871 detected in tumor-free mice partially overlapped with the peptide with the amino acid sequence of 267-273 and the $\mathrm{m} / \mathrm{z}$ of 790.429 found in mice with B16 melanoma. The peptide with the amino acid sequence of 280-294 and the $\mathrm{m} / \mathrm{z}$ of 1,666.8365 detected in tumor-free mice partially overlapped with the peptide with the amino acid sequence of 279-294 and the $\mathrm{m} / \mathrm{z}$ of $1,780.0200$ and completely overlapped with the peptide with the amino acid sequence of 280-289 and the $\mathrm{m} / \mathrm{z}$ ratio of $1,040.5248$ found in mice with B16 melanoma. The peptide with the amino acid sequence of 404-431 and the $\mathrm{m} / \mathrm{z}$ of $3,202.485$ detected in tumor-free mice partially overlapped with the peptide with the amino acid sequence of 404-424 and the $\mathrm{m} / \mathrm{z}$ of $2,354.1529$ from mice with B16 melanoma. The peptide with the amino acid sequence of 544-560 which was detected in tumor-free mice $(\mathrm{m} / \mathrm{z}, 2,156.1239)$ and in mice with B16 melanoma (m/z, 2,156.0816) partially overlapped with the peptide with the amino acids sequence of 547-566 and the $\mathrm{m} / \mathrm{z}$ of 2,226.2278 found in mice with B16 melanoma. The peptide with the amino acid sequence of 612-635 and the $\mathrm{m} / \mathrm{z}$ of $2,586.3754$ detected in tumor-free 
Table VI. Overlapping peptides of the inter-alpha-trypsin inhibitor heavy chain 4 in tumor-bearing and in tumor-free mice.

\begin{tabular}{|c|c|c|c|}
\hline Animals & $\begin{array}{l}\text { Peptide ion } \\
\qquad(\mathrm{m} / \mathrm{z})\end{array}$ & Start-end & Amino acid sequence \\
\hline Tumor-free mice & 1794.9294 & $104-121$ & K.QYSAAVGRGESAGIVKTT.G \\
\hline B16 melanoma & 916.5098 & $111-119$ & G.RGESAGIVK.T \\
\hline Tumor-free mice & 2415.1894 & $140-158$ & K.ITFELIYQELLQRRLGMYE.L \\
\hline B16 melanoma & 2544.3711 & $140-159$ & K.ITFELIYQELLQRRLGMYEL.L + oxidation (M) \\
\hline Tumor-free mice & 2943.3268 & $171-195$ & K.HLQMDIYIFEPQGISILETESTFMT.P \\
\hline Tumor-free mice & 1405.7547 & $171-181$ & K.HLQMDIYIFEP.Q \\
\hline B16 melanoma & 1405.7664 & & \\
\hline Tumor-free mice & 1016.4871 & $265-273$ & A.PENLPTMSK.N \\
\hline B16 melanoma & 790.429 & $267-273$ & E.NLPTMSK.N \\
\hline Tumor-free mice & 1666.8365 & $280-294$ & I.DKSGSMSGKKIQQTR.E + oxidation (M) \\
\hline B16 melanoma & 1780.0200 & $279-294$ & V.IDKSGSMSGKKIQQTR.E + oxidation (M) \\
\hline B16 melanoma & 1040.5248 & $280-289$ & I.DKSGSMSGKK.I + oxidation (M) \\
\hline Tumor-free mice & 3202.485 & $404-431$ & $\begin{array}{l}\text { R.EAINGQYSLFCLGFGFDVNYPFLEKMAL. } \\
\text { + oxidation }(\mathrm{M})\end{array}$ \\
\hline B16 melanoma & 2354.1529 & $404-424$ & R.EAINGQYSLFCLGFGFDVNYP.F \\
\hline Tumor-free mice & 2156.0816 & $544-560$ & F.MERLWALLTIQQQLEQR.I \\
\hline B16 melanoma & 2156.1239 & $544-560$ & F.MERLWALLTIQQQLEQR.I \\
\hline B16 melanoma & 2226.2278 & $547-566$ & R.LWALLTIQQQLEQRISASGA.E \\
\hline Tumor-free mice & 2586.3754 & $612-635$ & V.GDGMQRLPLAAQAHPFRPPVRGSK.L \\
\hline B16 melanoma & 3499.9311 & $610-641$ & V.EVGDGMQRLPLAAQAHPFRPPVRGSKLMTVLK.G \\
\hline Tumor-free mice & 1791.8798 & $656-673$ & R.ASRQYIPPGFPGPPGPPG.F \\
\hline B16 melanoma & 1987.0943 & $659-678$ & R.QYIPPGFPGPPGPPGFPAPP.G \\
\hline Tumor-free mice & 881.4796 & $822-827$ & T.EYKWKK.T \\
\hline B16 melanoma & 1330.7948 & $826-837$ & W.KKTLFSVLPGLK.M \\
\hline Tumor-free mice & 1311.651 & $895-905$ & V.WEPPVEPDNTK.R \\
\hline B16 melanoma & 1609.8461 & $896-909$ & W.EPPVEPDNTKRTVK.V \\
\hline Tumor-free mice & 793.4153 & $910-916$ & K.VQGVDYL.A \\
\hline B16 melanoma & 1277.697 & $906-916$ & K.RTVKVQGVDYL.A \\
\hline
\end{tabular}

M, methionine.

Table VII. Interaction between albumin and the fatty acid spin probe 16-doxyl stearate acid determined by electron spin resonance analysis.

\begin{tabular}{lccr}
\hline Parameter & Tumor-free mice & Tumor-bearing mice & P-value \\
\hline Discrimination parameter & $-3.08 \pm 0.06$ & $-2.2 \pm 0.09$ & $<0.05$ \\
Binding efficiency (\%) & $28.4 \pm 5.1$ & $22.0 \pm 5.0$ & $<0.05$ \\
Real transport quality (\%) & $39.9 \pm 5.4$ & $34.5 \pm 6.3$ & $>0.05$ \\
Detoxification efficiency (\%) & $14.6 \pm 4.5$ & $9.7 \pm 3.8$ & $>0.05$ \\
\hline
\end{tabular}

Data presented as the mean \pm standard deviation, $n>3$. Statistical significance calculated using Student's t-test.

mice partially overlapped with the peptide with the amino acid sequence of $610-641$ and the $\mathrm{m} / \mathrm{z}$ of 3,499.9311 found in mice with B16 melanoma. The peptide with the amino acid sequence 656-673 and the m/z of 1,791.8798 detected in tumor-free mice partially overlapped with the peptide with the amino acid sequence 659-678 and the $\mathrm{m} / \mathrm{z} 1,987.0943$ found in mice with B16 melanoma. The peptide with the amino acid sequence of $822-827$ and the $\mathrm{m} / \mathrm{z}$ of 881.4796 detected in tumor-free mice partially overlapped with the peptide with the amino acid sequence of 826-837 and the $\mathrm{m} / \mathrm{z}$ of 1,330.7948 found in mice with B16 melanoma. The peptide with the amino acid sequence of 895-905 and the $\mathrm{m} / \mathrm{z}$ of 1,311.651 detected in tumor-free mice partially overlapped with the peptide with the amino acid sequence of 896-909 


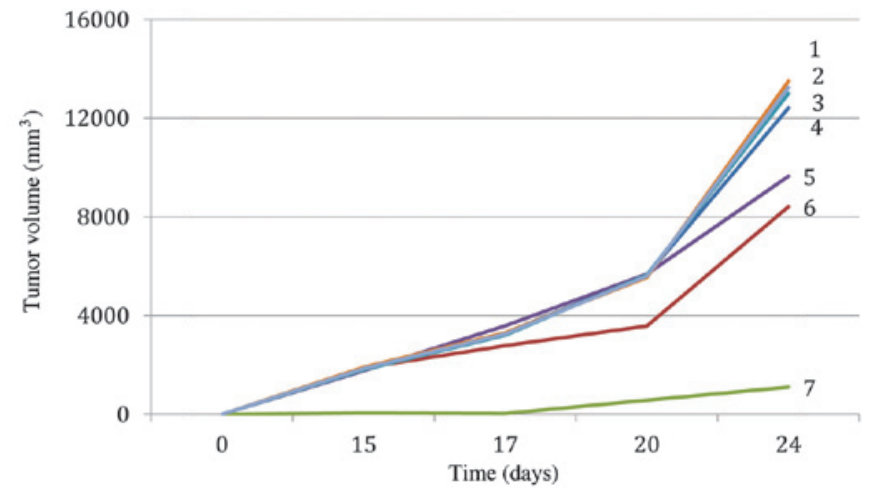

\begin{tabular}{rrrrrrrr}
\multicolumn{7}{c}{ Tumor volume $\left(\mathrm{mm}^{3}\right)$ in groups } \\
Day & 1 & 2 & 3 & 4 & 5 & 6 & 7 \\
0 & 0 & 0 & 0 & 0 & 0 & 0 & 0 \\
15 & 1900 & 1850 & 1800 & 1790 & 1765,2 & 1875,4 & 50 \\
17 & 3300 & 3250 & 3200 & 3300,8 & 3582,8 & 2782,4 & 29 \\
20 & 5550 & 5600 & 5650 & 5619,2 & 5679,6 & 3579,6 & 569 \\
24 & 13500 & 13250 & 13000 & 12413,25 & 9650,4 & 8413 & 1096
\end{tabular}

Figure 3. Influence of the inter-alpha-trypsin inhibitor heavy chain 4 and albumin on the growth of B16 melanoma in mice. The abscissa displays the days after transplantation of tumor cells and the ordinate displays the tumor volume ( $\mathrm{mm}^{3}$ ). Curves: (1) B16 melanoma growth in the control group; (2) B16 melanoma growth after injection of inter-alpha-trypsin inhibitor heavy chain 4, which was obtained from fresh blood serum of tumor-free C57Bl/6 mice; (3) B16 melanoma growth in mice after injection of albumin, obtained from fresh blood serum of C57Bl/6 mice with B16 melanoma; (4) B16 melanoma growth after injection of the animals with albumin obtained from fresh blood serum of tumor-free C57Bl/6 mice. (5) B16 melanoma growth after injection with inter-alpha-trypsin inhibitor heavy chain 4 obtained from frozen blood serum of C57Bl/6 mice with B16 melanoma. (6) B16 melanoma growth in mice after injection of inter-alpha-trypsin inhibitor heavy chain 4, which was obtained from the fresh blood serum of $\mathrm{F} 1$ hybrid mice $(\mathrm{CBAxC} 57 \mathrm{Bl} / 6)$ with $\mathrm{B} 16$ melanoma. (7) B16 melanoma growth in mice after injection of inter-alpha-trypsin inhibitor heavy chain 4, obtained from the fresh blood serum of C57B1/6 mice with B16 melanoma. Injections of proteins were performed two weeks prior to tumor cell inoculation. Student's t-test used for analysis and $\mathrm{n}=10$.

A

B

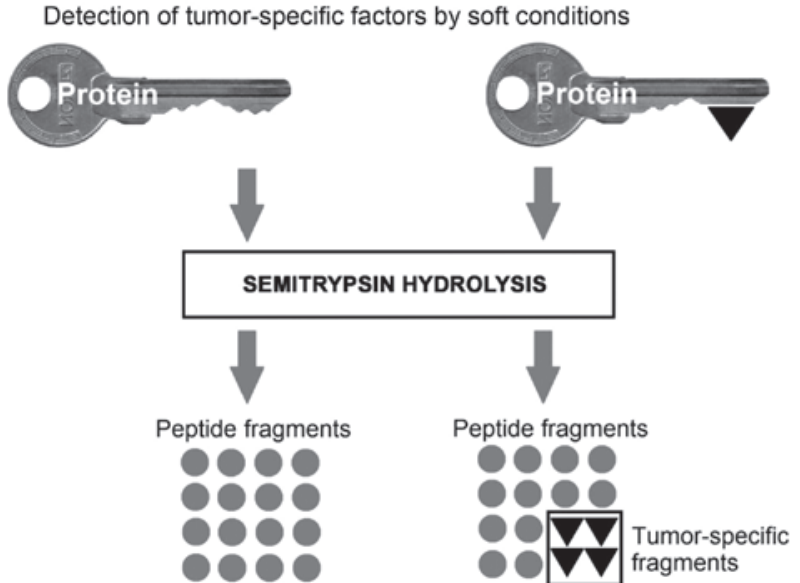

Figure 4. (A) Generation and (B) detection of tumor-specific factors.

and the $\mathrm{m} / \mathrm{z}$ of $1,609.8461$ found in mice with B16 melanoma. The peptide with the amino acid sequence of 910-916 and the $\mathrm{m} / \mathrm{z}$ of 793.4153 detected in tumor-free mice partially overlapped with the peptide with the amino acid sequence of 906-916 and the $\mathrm{m} / \mathrm{z}$ ratio of $1,277.697$ found in mice with B16 melanoma. Thus, the same pattern to that observed for albumin was confirmed for ITIH4, i.e. ITIH4 obtained from mice with B16 melanoma provided semi-tryptic peptide fragments that differed from the semi-tryptic peptide fragments of ITIH4 from the serum of tumor-free mice. This result indicated a difference in the availability of proteolysis sites and therefore, a different molecular conformation of albumin and ITIH4 in the serum of tumor-bearing and tumor-free mice.
Serum albumin has differential conformation-depending binding activity between tumor-bearing and tumor-free mice. In the next stage of the present study, it was required to test whether the biological activity of the assessed proteins was altered upon their conformational changes. For the assessment of the functional activity of albumin, ESR analysis was used, allowing for the estimation of the conformational status and transport parameters of albumin. For this purpose, the interaction of albumin with the spin probe 16-doxyl stearic acid was examined. As the results in Table VII demonstrate, the spin-labeled acid interacted differently with albumin depending on whether it was obtained from tumor-free or tumor-bearing mice. The largest difference (almost 1.4 times) among all values determined was observed in the discrimination parameter. 
According to the developers of the ESR device, this the discrimination parameter characterizes the conformation of the molecule. Therefore, the results obtained supported the hypothesis that albumin in tumor-bearing mice and tumor-free mice have differential conformations, which may be involved in the regulation of the tumor-associated activity of proteins.

Prior injection of ITIH4 from mice with B16 melanoma reduces melanoma growth in mice. Subsequently, a series of in vivo experiments were performed to study the influence of inter ITIH4 (serpin) and albumin on the growth of B16 melanoma (Fig. 3). For this purpose, mice were injected with albumin or ITIH4 from plasma from mice with B16 melanoma or tumor-free mice two weeks prior to tumor cell inoculation, and the effect on tumor growth was observed (4). It was discovered that albumin, regardless of cancer status of the animal from which it was obtained, had no effect on the growth of B16 melanoma in mice. Serpin obtained from fresh blood serum of tumor-free animals or from frozen blood serum of mice with B16 melanoma did not affect the growth of B16 melanoma. As shown in Fig. 3, a significant inhibition of the tumor growth was only achieved in group 7 , in which animals had been injected with ITIH4, which was obtained from fresh serum of C57Bl/6 mice with B16 melanoma, prior to transplantation of the tumor. Prior freezing of this serum or the use of allogeneic protein led to the loss of the tumor-specific activity. Thus, only the protein extracted from fresh blood serum of $\mathrm{C} 57 \mathrm{Bl} / 6$ mice significantly inhibited the growth of melanoma in $\mathrm{C} 57 \mathrm{Bl} / 6$ mice.

The inhibition of the tumor growth by ITIH4 protein was reflected by the lengthening of the period until the visual appearance of the tumor: In the mice of the control group, the appearance of the tumor was noted 10 days after tumor transplantation, whereas in the experimental group, the tumors were registered only at day 20 after tumor cell transplantation. This may be interpreted as $100 \%$ tumor growth inhibition by ITIH4 over 10 days. The lifespan of animals with B16 melanoma in the control group averaged $40.8 \pm 2.1$ days, whereas in group 7, in which ITIH4 was injected, it was $65.3 \pm 3.4$ days $(\mathrm{P}<0.05)$.

\section{Discussion}

To date, the biological effects of serum proteins and their regulation have not been fully elucidated. In the present study, the tumor-specific activity of serum proteins was assessed, as well as their regulation by conformational changes. However, the underlying mechanisms of this effect have yet to be elucidated. In addition, it was not possible to reproduce the in vivo results in an in vitro system; this may be due to the metabolism and homeostasis in a living organism, which cannot be reproduced by an in vitro cell model.

A previous study by our group demonstrated the tumor-specific activity of the blood serum fractions of mice with Ehrlich carcinoma, which contained proteins with a molecular weight of 50-100 kDa (4). This fraction contained 40 proteins and differences were most obvious by electrophoretic mobility assay in the major band with a molecular weight of $\sim 65 \mathrm{kDa}$. The major changes in this band coincided with the disappearance of alpha-1-anti-trypsin and the appearance in the same sample of cathepsin L1. Inter- $\alpha$-trypsin inhibitor was additionally identificated in this fraction. This led to the hypothesis that the tumor-specific activity of serpin is exerted by $\alpha$-1-anti-trypsin, even though the molecular weight of this protein is significantly lower and was $45 \mathrm{kDa}$. In the present study, a protein with a similar molecular weight of 65-70 kDa from the serum of mice with B16 melanoma, identified as serpin ITIH4, also showed a specific activity. The results of the previous studies by our group as well as those obtained in the present study suggested that in the two cases - Ehrlich carcinoma and B16 melanoma - the protein with a tumor-specific activity was identical and that its conformation determined its biological specificity.

In previous studies, our group as well as other researchers, did not identify any significant differences in the spectrum of proteins isolated from the serum of tumor-bearing and tumor-free mice $(3,4)$. In spite of this, these blood serum proteins were observed to have a tumor-specific activity. This fact can be explained by differences in sample preparation of these proteins in the previous and present studies. In particular, in prior studies, at least two methods were used which cause the denaturation of proteins (prior freezing of samples or boiling in a buffer for electrophoresis). The denaturation facilitates proteolysis and the identification of proteins, but prevents the evaluation of features of their conformation.

Therefore, in the present study, the protein conformation was not investigated using these standard methods. Only fresh serum samples were used, the separation of proteins was performed by column chromatography and the changes in conformation of the proteins were estimated via the soft proteolysis (with $10 \%$ acetonitrile) product. It was discovered that identical sites of the same protein yielded differential peptide fragment spectra between tumor-free mice and those with melanoma. This result demonstrated the difference in the availability of these sites for the enzymes, and hence, the differential conformation of the proteins between the two experimental groups. It is important to note that the conformational changes of the proteins studied were associated with changes in their biological activity. Thus, the present study demonstrated for the first time, to the best of our knowledge, that the tumor-specific activity of ITIH4 was based on its differential conformation between tumor-free mice and those with melanoma. This result confirmed the hypothesis that the conformation of serpin determines its tumor-specific effect.

The results obtained can be figuratively explained as illustrated in the scheme in Fig. 4A: Serum protein can be regarded as a key, which has a stable part and a specific component. The stable part, represented in the present study by common peptides, is present in all organisms of the given species. In the present study, the specific component is constituted by unique peptides in a particular individual and is defined by its state, in particular, by the presence of a tumor. The main condition for the functioning of this protein (key) is the lack of high affinity to the tissues of the body and other serum proteins. Tumor growth leads to an increased concentration of these groups of antigenic determinants, which are located on the surface of the tumor cells. In response to this increased concentration of groups of antigenic determinants in cells, the availability of antigenic determinants, which are capable of interacting with them, 
are expected to decrease in the serum. This may be achieved in two ways: The emergence of acute phase proteins among the blood serum proteins (5), and conformational changes in serum proteins, as shown in the present study. In order to study this adaptation to tumor growth, the present study performed a proteomics study, which required proteases to cleave the native protein. Conventional methods of proteomic analyses of proteins include the preliminary freezing of the samples and boiling for electrophoresis. However, in the present study, this denaturation resulted in the loss of the tumor-specific activity of the samples. Therefore, the conditions of sample preparation were modified by excluding any experimental conditions leading to protein denaturation (Fig. 4B).

In conclusion, the results of the present study confirmed the hypothesis that the conformation of serum proteins is associated with their biological activity and with tumor growth in the body. Injection of ITIH4 from the serum of mice with melanoma was demonstrated to inhibit tumor growth in a mouse model of melanoma under the condition that its conformation was preserved.

\section{References}

1. Donenko FV, Ziganshin RK, Sitdikova SM, Amandzholov BS, Kiselevskii MV and Efferth T: Induction of resistance to murine tumor development is associated with alterations in the glycosylation of blood serum proteins. Mol Med Rep 2: 487-495, 2009. doi: $10.3892 / \mathrm{mmr} 00000126$.

2. Donenko FV, Kabieva AO and Efferth T: Tumor-specific blood serum factors as determinants of tumor growth. Klin Lab Diagn 50-52, 13-15, 2013 (In English, Russian).

3. Kormosh NG, Ziganshin RKh, Shender VO, Voyushin KE and Donenko FV: Changes in the serum protein composition in mice with transplanted Ehrlich's carcinoma. Bull Exp Biol Med 158 489-492, 2015

4. Donenko FV, Ziganshin RH, Anisimova NY, Voyushin KE Sitdikova SM, Amandzholov BS, Kiselevskii MV and Efferth T: Identification of serpin (alpha-1-antitrypsin) as serum growth inhibitory factor in murine ehrlich carcinoma by proteomics. Cancer Genomics Proteomics 7: 147-156, 2010.
5. Fisher B, Gunduz N, Coyle J, Rudock C and Saffer E: Presence of a growth-stimulating factor in serum following primary tumor removal in mice. Cancer Res 49: 1996-2001, 1989.

6. Sitdikova SM, Amandzholov BS, Kiselevskii MV and Donenko FV: Specificity of relapses and metastases of experimental transplanted Ehrlich carcinoma and B16 melanoma. Bull Exp Biol Med 143: 80-82, 2007.

7. Kondo T: Inconvenient truth: Cancer biomarker development by using proteomics. Biochim Biophys Acta 1844: 861-865, 2014.

8. Sitdikova SM, A mandzholov BS, Kiselevskii MV and Donenko FV: Lectin binding to mouse blood lymphocytes during tumor growth. Bull Exp Biol Med 140: 445-448, 2005.

9. Donenko FV, Kabieva AO, Volkov IuT and Moroz LV: Mouse serum inhibition of cytotoxicity of goat antibodies against mouse thymocytes. Biull Eksp Biol Med 113: 642-644, 1992 (In Russian).

10. Donenko FV, Sitdikova SM, Syrtsev AV, Gradyushko AT, Kiselevsky MV, Serebryakova MV and Efferth T: Hemoglobin-associated proteins isolated from blood serum of Ehrlich carcinoma-bearing mice. Int J Oncol 32: 885-893, 2008.

11. Sergeant K, Pinheiro C, Hausman JF, Ricardo CP and Renaut J: Taking advantage of nonspecific trypsin cleavages for the identification of seed storage proteins in cereals. J Proteome Res 8: 3182-3190, 2009. doi: 10.1021/pr801093f.

12. Alves G and Yu YK: Improving peptide identification sensitivity in shotgun proteomics by stratification of search space. J Proteome Res 12: 2571-2581, 2013.

13. Law RH, Zhang Q, McGowan S, Buckle AM, Silverman GA, Wong W, Rosado CJ, Langendorf CG, Pike RN, Bird PI and Whisstock JC: An overview of the serpin superfamily. Genome Biol 7: 216, 2006.

14. Goldin A, Kline I and Sofina ZP (eds): Experimental Evaluation of Antitumor Drugs in the USA and USSR and Clinical Correlations. National Cancer Institute Monograph 55. NIH Publication no. 1933. U.S. Department of Health and Human Services. National Institutes of Health, NCI, Bethesda, MD, 1980.

15. Laemmli UK: Cleavage of structural proteins during the assembly of head of bacteriophage T4. Nature 227: 680-685, 1970.

16. Gurachevsky A, Shimanovitch E, Gurachevskaya T and Muravsky V: Intra-albumin migration of bound fatty acid probed by spin label ESR. Biochem Biophys Res Commun 360: 852-856, 2007.

17. Dickneite G, Herwald H, Korte W, Allanore Y, Denton CP and Matucci Cerinic M: Coagulation factor XIII: A multifunctional transglutaminase with clinical potential in a range of conditions. Thromb Haemost 113: 686-697, 2015. 